Dielectric Behavior of Reaction Mixture during Acid-Catalyzed Transesterification Reactions in

\title{
Biodiesel Production
}

Eduardo G. R. Taques Filho ${ }^{a}$, Evandro L. Dall'Oglio ${ }^{a^{*}}$, Paulo T. de Sousa Jr. ${ }^{\text {a }}$,Leonardo Gomes de Vasconcelos, Carlos Alberto Kuhnen ${ }^{\text {b }}$

a Departamento de Química Universidade Federal do Mato Grosso Av. Fernando Corrêa da Costa s/n, Coxipó,Cuiabá - MT, CEP:78090600; ${ }^{b}$ Departamento de Física - Universidade Federal do Santa Catarina; Campus Universitário Trindade Florianópolis-SC - CEP 88040970

\section{Supporting Information}

\section{Summary}

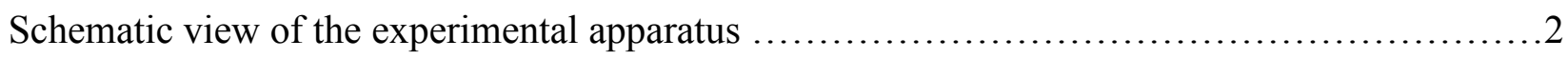

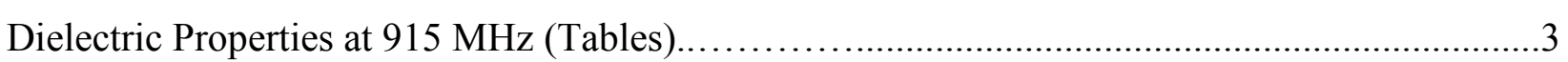

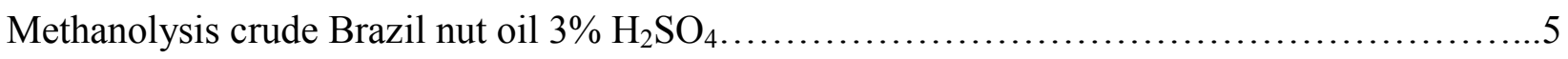

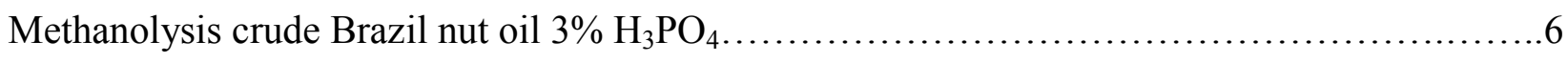

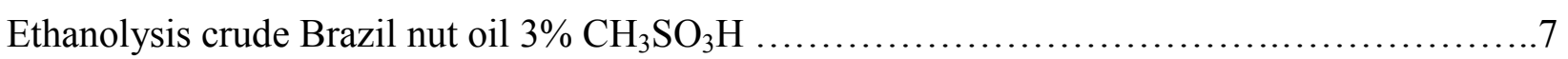

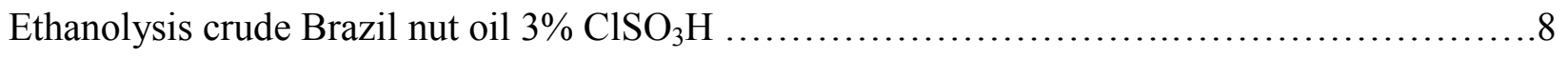

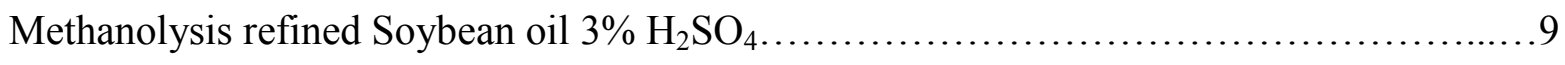

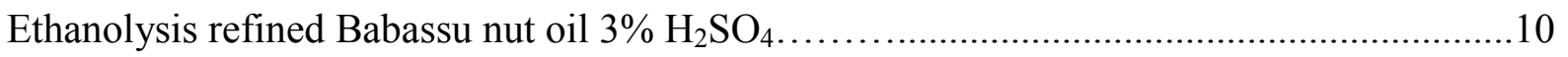

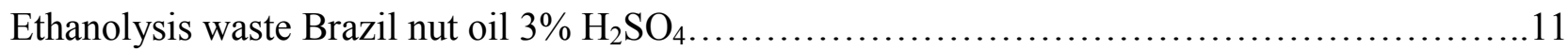

\section{Comments concerning homogeneity}

It should be noted that the measurement of the dielectric constant and loss factor of reacting mixtures during ethanolysis performed with methanesulfonic and chlorosulfonic presents difficulties related to the oil/ethanol molar ratio used (1:6) due to the lack in the homogeneity of the initial mixture with the addition of $3 \%$ $\left(\mathrm{V} / \mathrm{V}_{\mathrm{T}}\right)$ of these acids. In the same manner, the reaction mixtures in the ethanolysis of refined babassu and waste Brazil nut oils employing sulfuric acid exhibit difficulties in relation to the homogenization with the addition of acids in the initial oil/ethanol mixture and with the formation of glycerol during ethanolysis. 


\section{FIGURE S1}

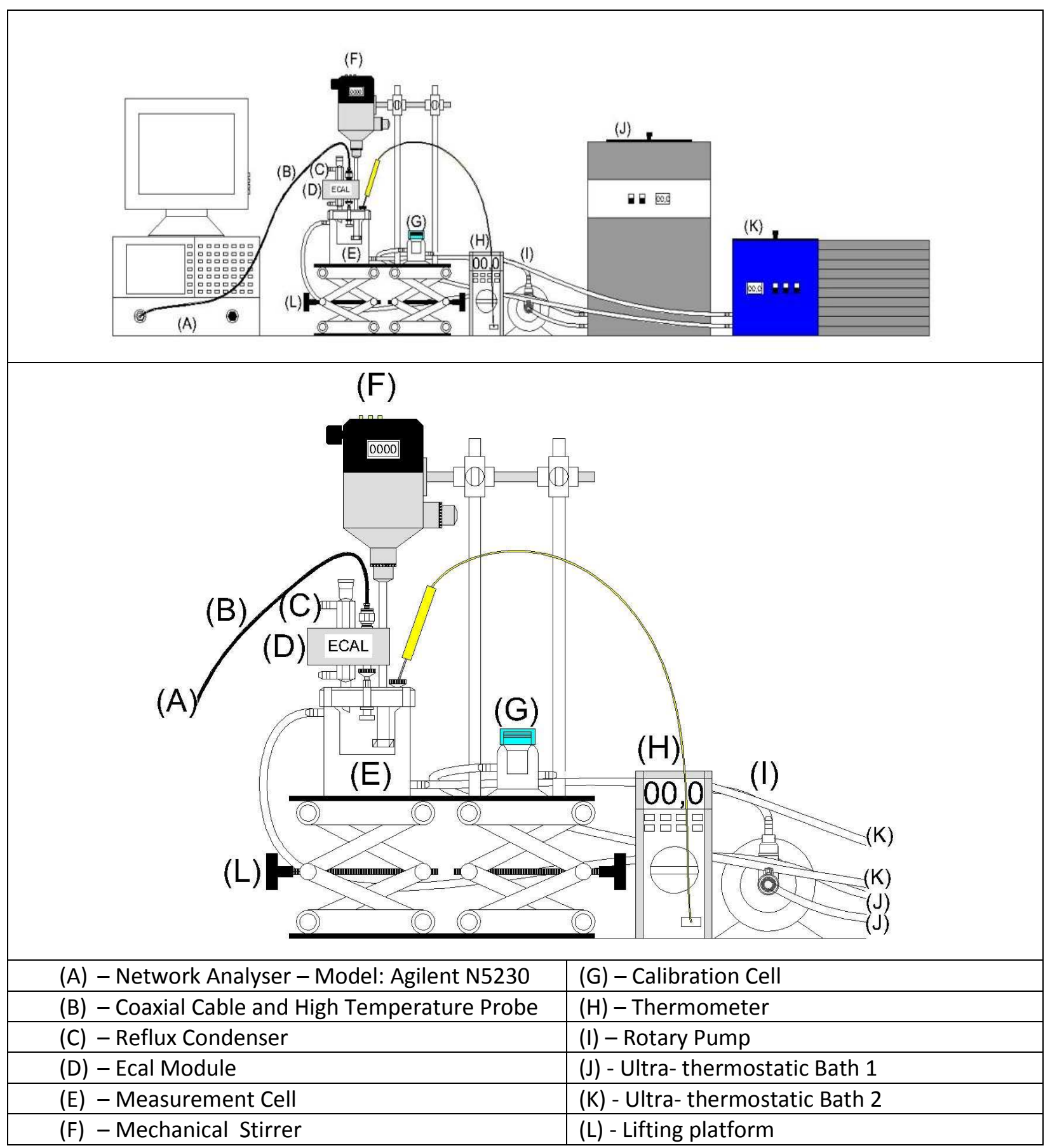

Figure S1. Schematic view of the experimental apparatus 
Table S1

\begin{tabular}{|c|c|c|c|c|c|c|c|}
\hline \multicolumn{2}{|c|}{ Brazil nut oil } & \multicolumn{6}{|c|}{ Frequency 915MHz } \\
\hline \multirow{2}{*}{ Alcohol } & \multirow{2}{*}{ Acid } & \multicolumn{3}{|c|}{ Initial } & \multicolumn{3}{c|}{ Final } \\
\cline { 3 - 8 } & $\varepsilon^{\prime}$ & $\varepsilon^{\prime}{ }_{\text {ef }}$ & Tg $\delta$ & $\varepsilon^{\prime}$ & $\varepsilon^{\prime}{ }_{\text {ef }}$ & Tg $\delta$ \\
\hline \multirow{4}{*}{$\begin{array}{c}\text { Methan } \\
\text { ol }\end{array}$} & $\mathrm{H}_{2} \mathrm{SO}_{4}$ & 5.52 & 1.46 & 0.26 & 3.68 & 0.55 & 0.15 \\
\cline { 2 - 8 } & $\mathrm{H}_{3} \mathrm{PO}_{4}$ & 4.73 & 0.75 & 0.16 & 4.69 & 0.96 & 0.20 \\
\cline { 2 - 8 } & $\mathrm{ClSO}_{3} \mathrm{H}$ & 5.22 & 1.20 & 0.23 & 7.71 & 4.17 & 0.54 \\
\cline { 2 - 8 } & $\mathrm{CH}_{3} \mathrm{SO}_{2} \mathrm{OH}$ & 5.46 & 1.19 & 0.21 & 4.82 & 4.29 & 0.89 \\
\hline \multirow{4}{*}{ Ethanol } & $\mathrm{H}_{2} \mathrm{SO}_{4}$ & 5.69 & 1.38 & 0.24 & 4.96 & 0.41 & 0.08 \\
\cline { 2 - 8 } & $\mathrm{H}_{3} \mathrm{PO}_{4}$ & 4.89 & 0.41 & 0.08 & 5.70 & 1.19 & 0.21 \\
\cline { 2 - 8 } & $\mathrm{ClSO}_{3} \mathrm{H}$ & 4.80 & 1.51 & 0.31 & 4.29 & 1.06 & 0.25 \\
\cline { 2 - 8 } & $\mathrm{CH}_{3} \mathrm{SO}_{2} \mathrm{OH}$ & 5.40 & 1.10 & 0.20 & 7.15 & 1.82 & 0.25 \\
\hline
\end{tabular}

Table S1. Dielectric parameters, at $915 \mathrm{MHz}$, in the beginning and in the end of transesterification reaction of refined Brazil nut oil employing methanol and ethanol and four different acids.

Table S2

\begin{tabular}{|c|c|c|c|c|c|c|c|c|}
\hline $\begin{array}{c}\text { Penetration Depth } \\
\text { at 915MHz (cm) }\end{array}$ & \multicolumn{2}{|c|}{$\mathrm{H}_{2} \mathrm{SO}_{4}$} & \multicolumn{2}{c|}{$\mathrm{H}_{3} \mathrm{PO}_{4}$} & \multicolumn{2}{c|}{$\mathrm{ClSO}_{3} \mathrm{H}$} & \multicolumn{2}{c|}{$\mathrm{CH}_{3} \mathrm{SO}_{2} \mathrm{OH}$} \\
\hline & & \multicolumn{2}{|c|}{} & & & \\
\hline Alcohol & Initial & Final & Initial & Final & Initial & Final & Initial & Final \\
\hline Methanol & 10.10 & 26.59 & 19.56 & 15.40 & 12.25 & 3.53 & 12.35 & 3.44 \\
\hline Ethanol & 10.66 & 35.74 & 35.54 & 12.39 & 9.78 & 13.86 & 13.42 & 8.11 \\
\hline
\end{tabular}

Table S2. Penetration depths, at $915 \mathrm{MHz}$, in the beginning and in the end of transesterification reaction of refined Brazil nut oil employing methanol and ethanol and four different acids as catalysts. 
Table S3

\begin{tabular}{|c|c|c|c|c|c|c|c|}
\hline \multicolumn{2}{|c|}{ Acid: $\mathrm{H}_{2} \mathrm{SO}_{4}$} & \multicolumn{6}{|c|}{ Frequency $915 \mathrm{MHz}$} \\
\hline \multirow{2}{*}{ Alcohol } & \multirow{2}{*}{ Oil } & \multicolumn{3}{|c|}{ Initial } & \multicolumn{3}{c|}{ Final } \\
\cline { 3 - 8 } & & $\varepsilon^{\prime}$ & $\varepsilon^{\prime}{ }_{\text {ef }}$ & Tg $\delta$ & $\varepsilon^{\prime}$ & $\varepsilon^{\prime}{ }_{\text {ef }}$ & Tg $\delta$ \\
\hline \multirow{4}{*}{ Methanol } & Refined Brazil nut & 5.51 & 1.46 & 0.26 & 3.68 & 0.55 & 0.15 \\
\cline { 2 - 8 } & Soybean & 5.93 & 1.41 & 0.23 & 5.13 & 1.03 & 0.20 \\
\cline { 2 - 8 } & Babassu & 6.19 & 1.62 & 0.26 & 5.72 & 2.39 & 0.42 \\
\cline { 2 - 8 } & Waste Brazil nut & 4.48 & 0.65 & 0.14 & 5.09 & 1.70 & 0.33 \\
\hline \multirow{4}{*}{ thanol } & Refined Brazil nut & 5.69 & 1.38 & 0.24 & 4.96 & 0.41 & 0.08 \\
\cline { 2 - 8 } & Soybean & 5.34 & 1.51 & 0.28 & 5.68 & 1.95 & 0.34 \\
\cline { 2 - 8 } & Babassu & 5.75 & 1.43 & 0.25 & 5.76 & 0.76 & 0.13 \\
\cline { 2 - 8 } & Waste Brazil nut & 5.49 & 1.20 & 0.21 & 6.16 & 0.83 & 0.13 \\
\hline
\end{tabular}

Table S3. Dielectric parameters, at $915 \mathrm{MHz}$, in the beginning and in the end of transesterification reaction of different oils employing methanol and ethanol and $\mathrm{H}_{2} \mathrm{SO}_{4}$ as catalyst.

Table S4

\begin{tabular}{|c|c|c|c|c|c|c|c|c|}
\hline $\begin{array}{c}\text { Penetration } \\
\begin{array}{c}\text { Depth at 915 } \\
\text { MHz }(\mathrm{cm})\end{array}\end{array}$ & \multicolumn{2}{|c|}{ Refined Brazil nut } & \multicolumn{2}{|c|}{ Soybean } & \multicolumn{2}{c|}{ Babassu } & \multicolumn{2}{c|}{ Waste Brazil nut } \\
\hline Alcohol & Initial & Final & Initial & Final & Initial & Final & Initial & Final \\
\hline Methanol & 10.10 & 26.59 & 10.47 & 14.28 & 9.10 & 6.16 & 22.47 & 8.64 \\
\hline Ethanol & 10.66 & 35.74 & 9.75 & 7.57 & 10.08 & 19.27 & 12.30 & 17.70 \\
\hline
\end{tabular}

Table S4. Penetration depths, at $915 \mathrm{MHz}$, in the beginning and in the end of transesterification reaction of different oils employing methanol, ethanol and $\mathrm{H}_{2} \mathrm{SO}_{4}$ as catalyst. 
Figure S2
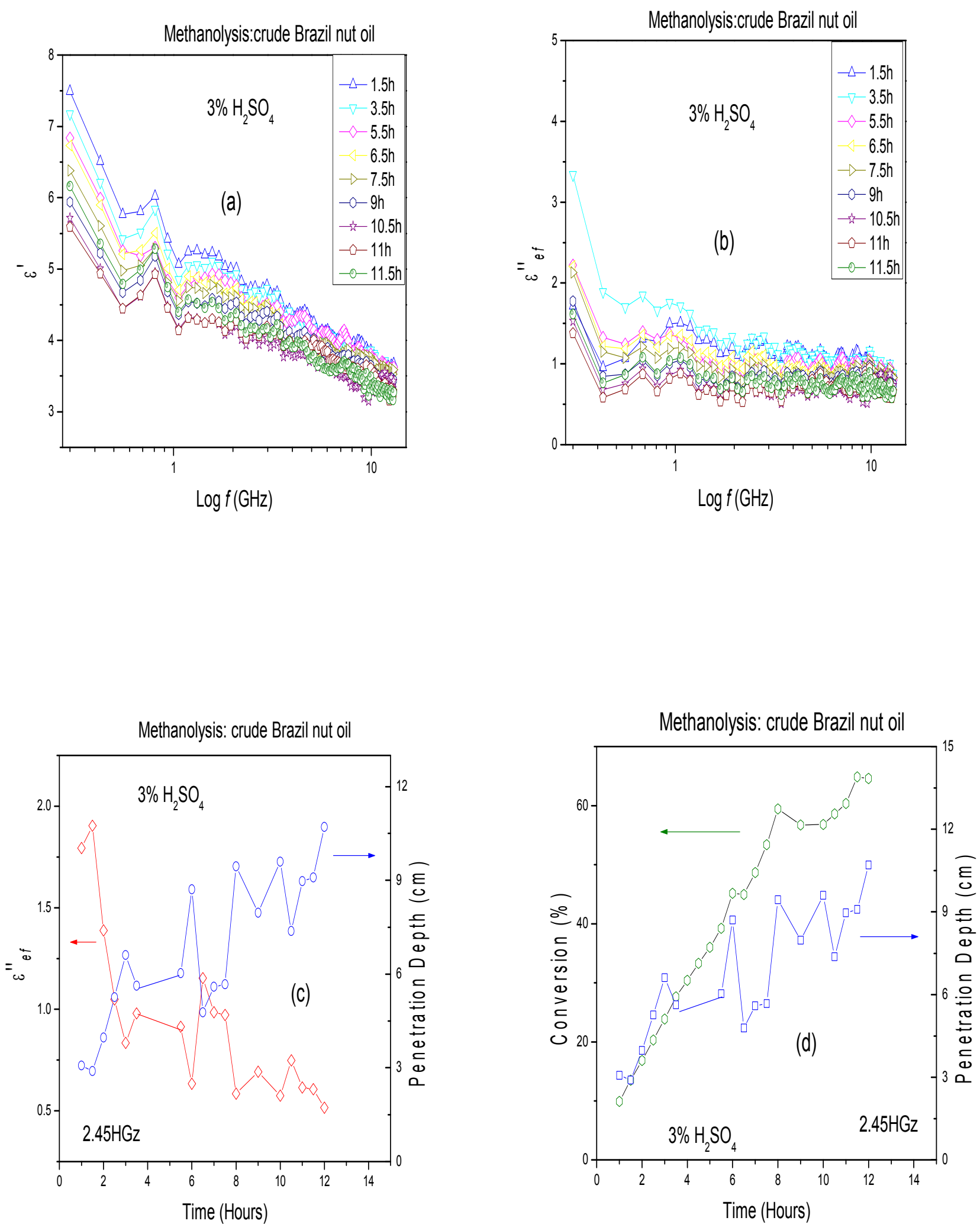

Figure S2. (a) Relative dielectric constant, $\varepsilon^{\prime}$, and (b) relative dielectric loss, $\varepsilon_{\mathrm{ef}}{ }^{\prime \prime}$; (c) relative dielectric loss and Penetration depth at $2.45 \mathrm{GHz}$; (d) Conversion to Biodiesel and Penetration depth at $2.45 \mathrm{GHz}$ during acid-catalyzed methanolysis of crude Brazil nut oil. Acid concentration: 3\% (V/VT) $\mathrm{H}_{2} \mathrm{SO}_{4}$; reaction temperature, $60^{\circ} \mathrm{C}$. 


\section{Figure S3}
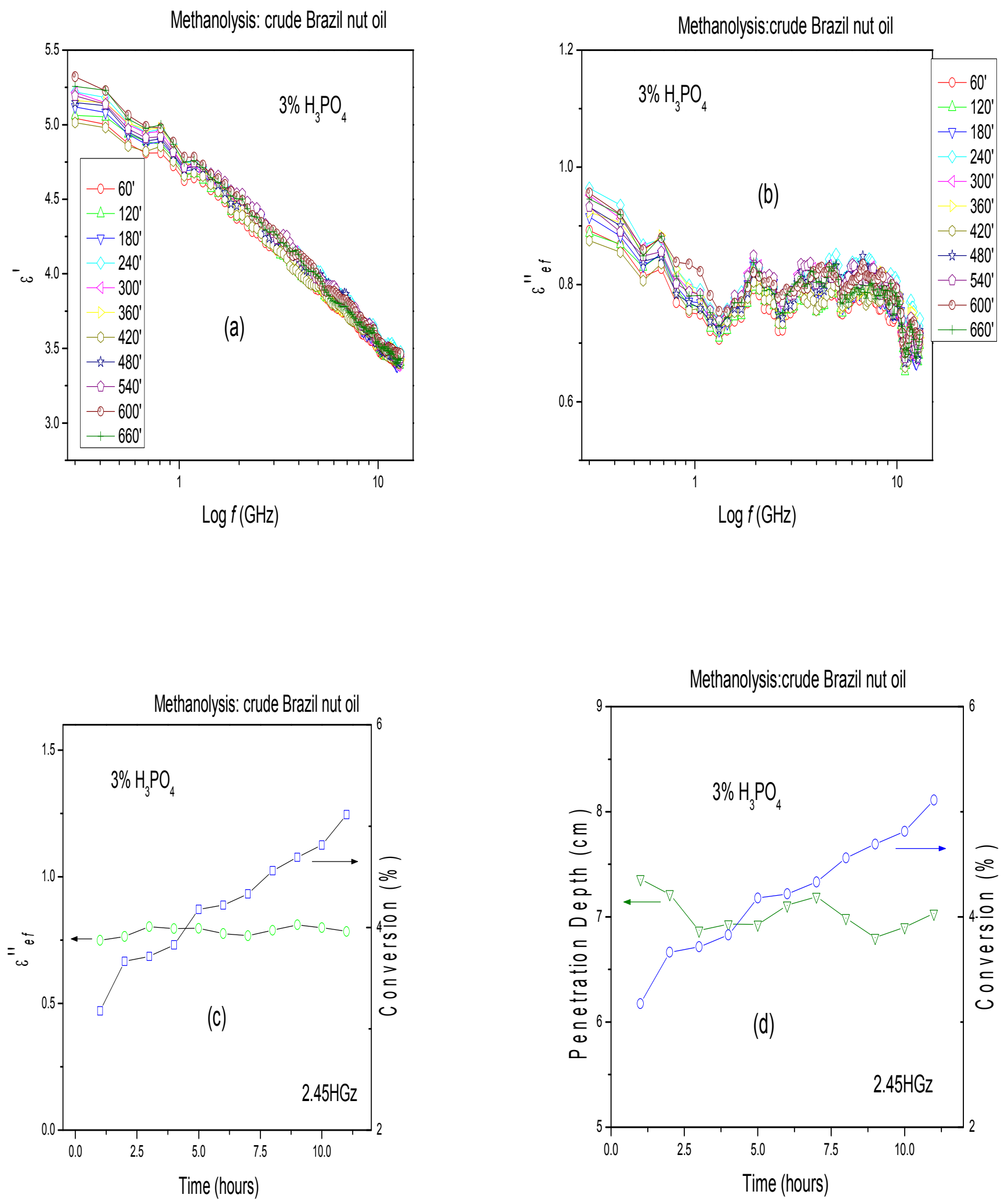

Figure S3. (a) Relative dielectric constant, $\varepsilon^{\prime}$, and (b) relative dielectric loss, $\varepsilon_{\mathrm{ef}}^{\prime \prime}$; (c) relative dielectric loss at 2.45 GHz and Conversion to Biodiesel; (d) Penetration depth at $2.45 \mathrm{GHz}$ and Conversion to Biodiesel during acid-catalyzed methanolysis of crude Brazil nut oil. Acid concentration: 3\% (V/VT) $\mathrm{H}_{3} \mathrm{PO}_{4}$; reaction temperature, $60^{\circ} \mathrm{C}$. 


\section{Figure S4}
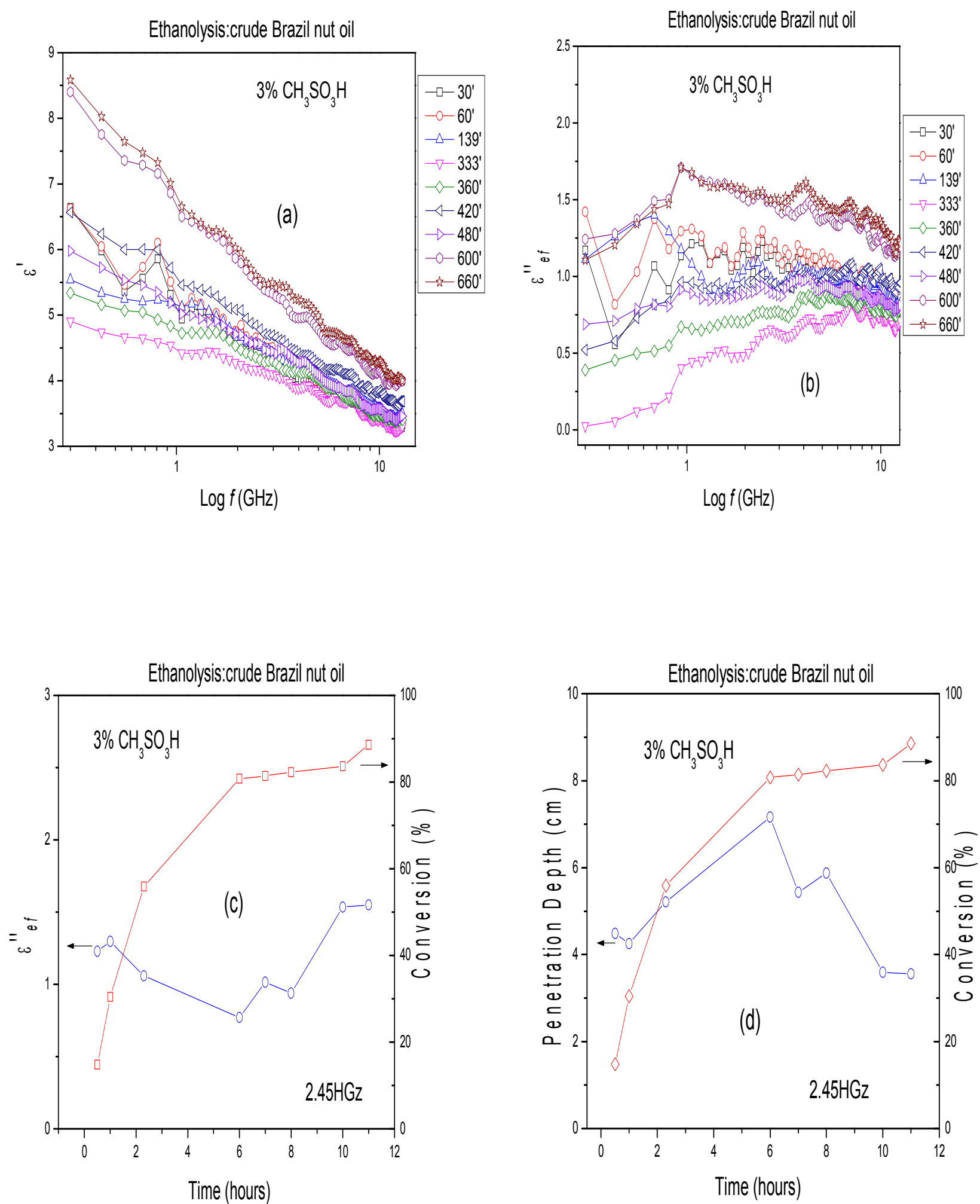

Figure S4. (a) Relative dielectric constant, $\varepsilon^{\prime}$, and (b) relative dielectric loss, $\varepsilon_{\mathrm{ef}}$ "; (c) relative dielectric loss at 2.45 GHz and Conversion to Biodiesel; (d) Penetration depth at $2.45 \mathrm{GHz}$ and Conversion to Biodiesel during acid-catalyzed ethanolysis of crude Brazil nut oil. Acid concentration: $3 \%(\mathrm{~V} / \mathrm{VT}) \mathrm{CH}_{3} \mathrm{SO}_{3} \mathrm{H}$; reaction temperature, $70^{\circ} \mathrm{C}$. 
Figure S5
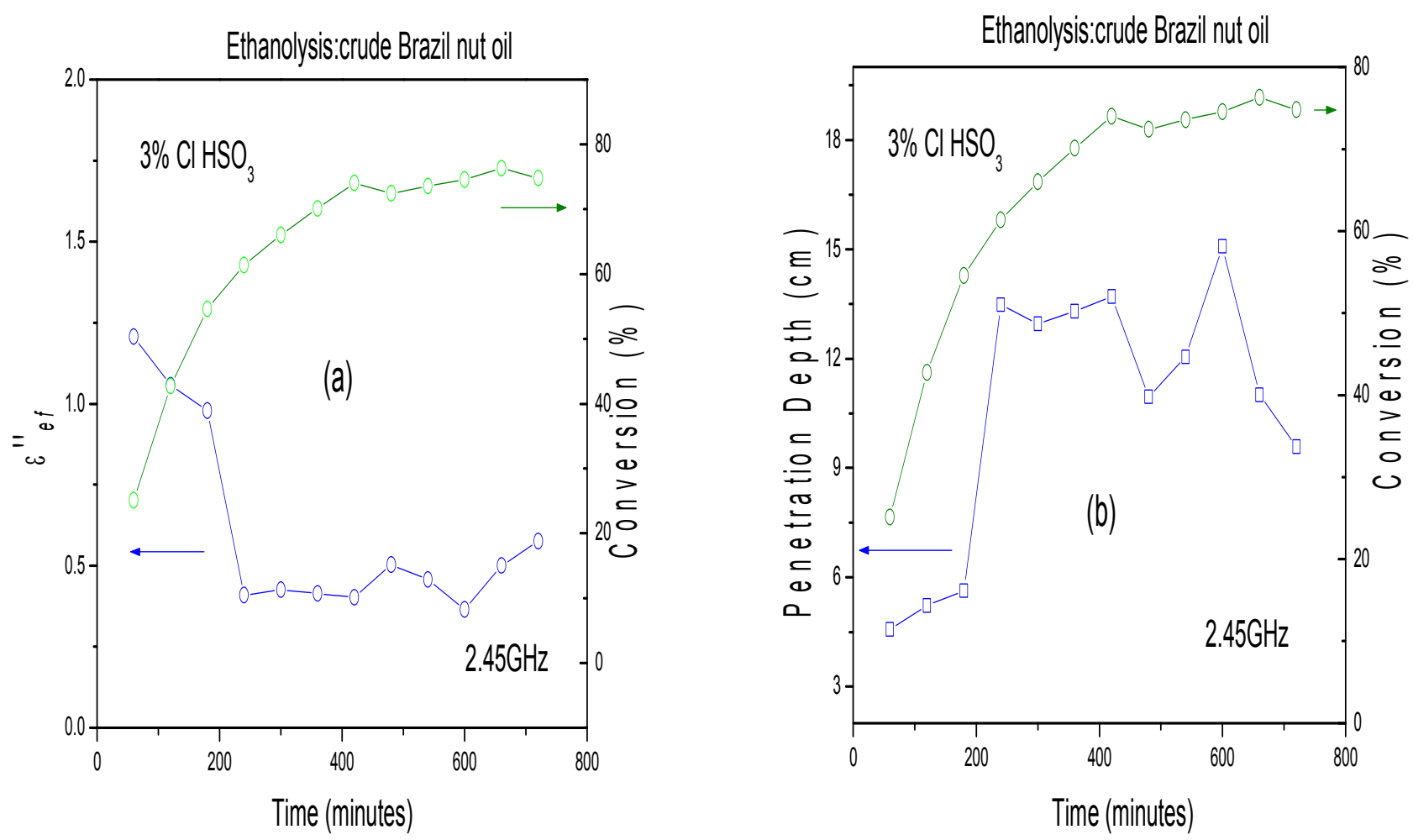

Figure S5. (a) Relative dielectric loss, $\varepsilon_{\mathrm{ef}}{ }^{\prime \prime}$, at $2.45 \mathrm{GHz}$ and Conversion to Biodiesel; (b) Penetration depth at 2.45 GHz and Conversion to Biodiesel during acid-catalyzed ethanolysis of crude Brazil nut oil. Acid concentration: $3 \%(\mathrm{~V} / \mathrm{VT}) \mathrm{ClHSO}_{3}$; reaction temperature, $70^{\circ} \mathrm{C}$. 


\section{Figure S6}
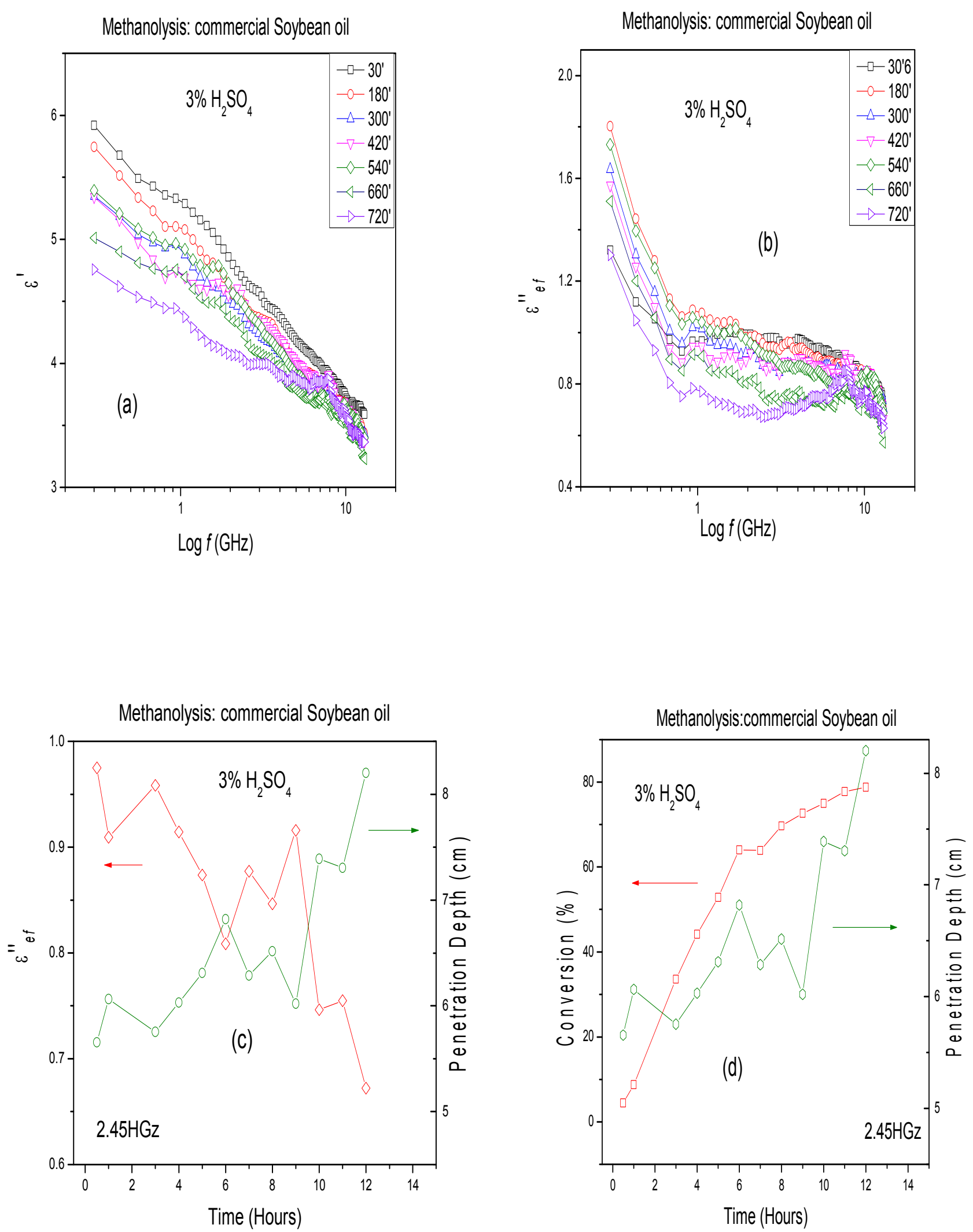

Figure S6. (a) Relative dielectric constant, $\varepsilon^{\prime}$, and (b) relative dielectric loss, $\varepsilon_{\mathrm{ef}}$ "; (c) relative dielectric loss and Penetration depth at $2.45 \mathrm{GHz}$; (d) Conversion to Biodiesel and Penetration depth at $2.45 \mathrm{GHz}$ during acid-catalyzed methanolysis of refined Soybean oil. Acid concentration: $3 \%(\mathrm{~V} / \mathrm{VT}) \mathrm{H}_{2} \mathrm{SO}_{4}$; reaction temperature, $60^{\circ} \mathrm{C}$. 
Figure S7
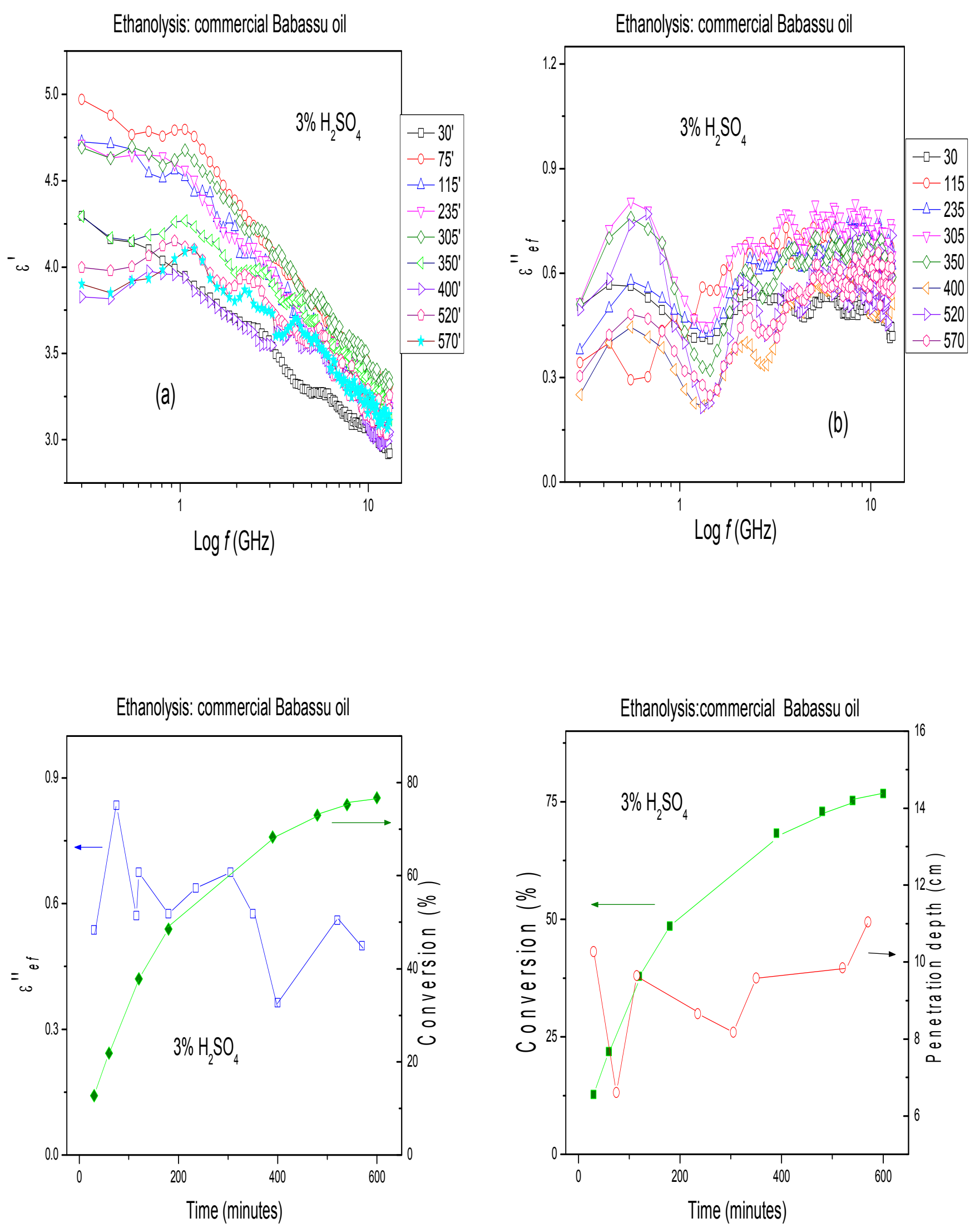

Figure S7. (a) Relative dielectric constant, $\varepsilon^{\prime}$, and (b) relative dielectric loss, $\varepsilon_{\mathrm{ef}}^{\prime \prime}$; (c) relative dielectric loss at $2.45 \mathrm{GHz}$ and Conversion to Biodiesel; (d) Conversion to Biodiesel and Penetration depth at $2.45 \mathrm{GHz}$ during acid-catalyzed ethanolysis of refined Babassu oil. Acid concentration: 3\% (V/VT) $\mathrm{H}_{2} \mathrm{SO}_{4}$; reaction temperature, $70^{\circ} \mathrm{C}$. 


\section{Figure S8}
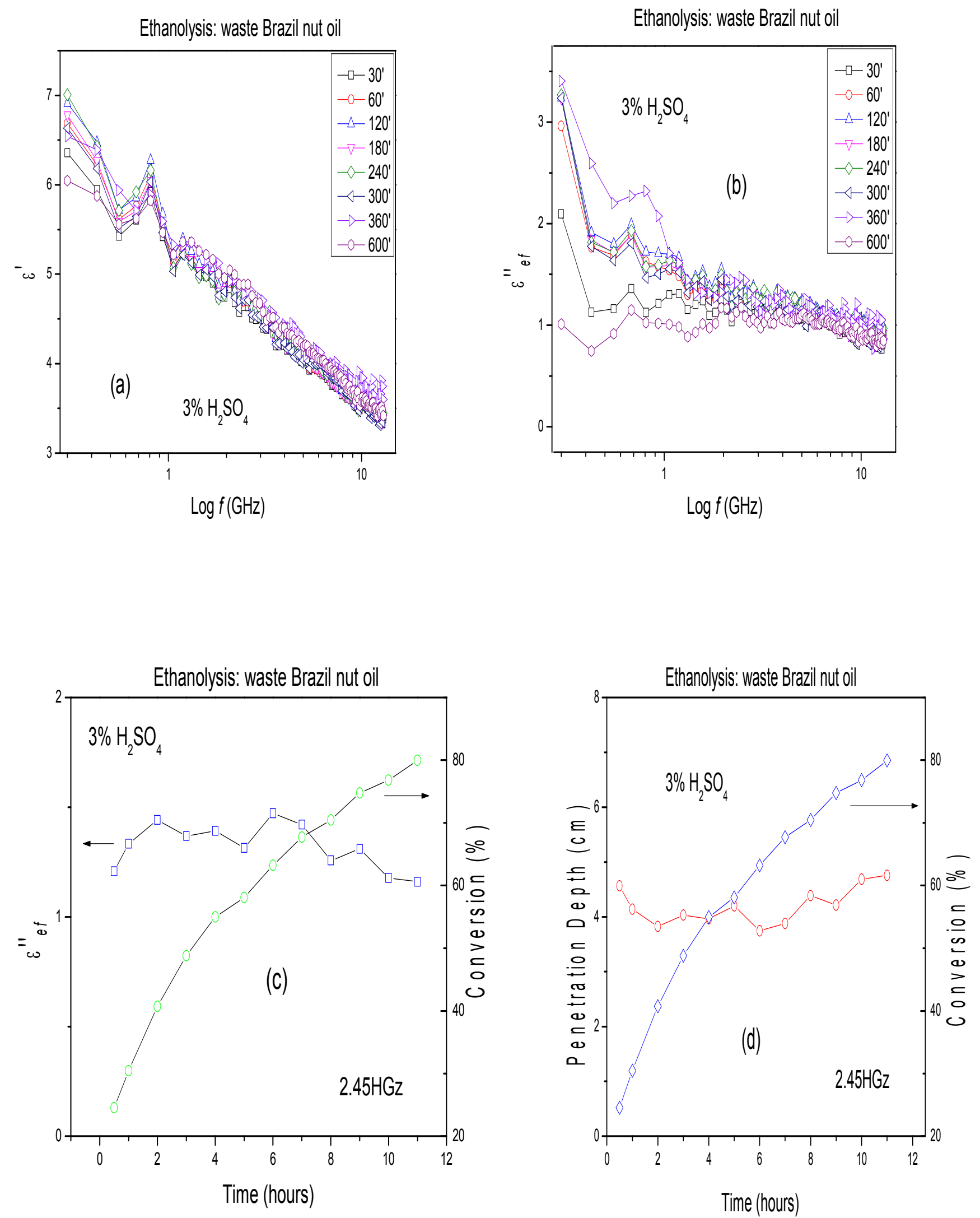

Figure S8. (a) Relative dielectric constant, $\varepsilon^{\prime}$, and (b) relative dielectric loss, $\varepsilon_{\mathrm{ef}}^{\prime \prime}$; (c) relative dielectric loss at $2.45 \mathrm{GHz}$ and Conversion to Biodiesel; (d) Penetration depth at $2.45 \mathrm{GHz}$ and Conversion to Biodiesel during acid-catalyzed ethanolysis of waste Brazil nut oil. Acid concentration: 3\% (V/VT) $\mathrm{H}_{2} \mathrm{SO}_{4}$; reaction temperature, $70^{\circ} \mathrm{C}$. 